\title{
Unbalanced and Double Line to Ground Fault Detection of Three Phase VSI Fed Induction Motor Drive using Fuzzy Logic Approach
}

\author{
K.Mohanraj \\ Asst.Professor \\ EEE Department \\ SRM University, Chennai
}

\author{
Sridhar Makkapati \\ PG Scholar \\ EEE Department \\ SRM University, Chennai
}

\author{
S.Paramasivam, Ph.D \\ Research Head \\ ESAB \\ Chennai, India
}

\begin{abstract}
Online monitoring of induction motors is becoming increasingly important. Knowledge based fuzzy logic approach helps in diagnosing the induction motor faults. The current work presents an effective method for diagnosing the stator side faults such as double line fault and an unbalance fault. Stator condition is diagnosed based on the stator RMS values of current amplitude in addition to the knowledge expressed in rules and membership function. The model is implemented in MATLAB/SIMULINK with the data obtained under both healthy and faulty condition and has ability to work with variable speed drives.
\end{abstract}

\section{General Terms}

Fuzzy logic, Fuzzy inference, Rule viewer.

\section{Keywords}

Diagnosis, Fuzzy logic, Unbalance, Stator current amplitude, Knowledge base.

\section{INTRODUCTION}

Three phase induction motors are work horses in many of the industrial applications because of its simple structure and reliability. In an industrial application these motors are utilized in between $40 \%$ to $50 \%$ in wide range applications. However, these machines owe due to the thermal, electrical and mechanical stresses which are unavoidable. Early detection of these abnormal disturbances helps in avoiding the severe failures in the system. This helps the electrical drive system to reduce maintenance cost and unscheduled downtimes which impact more on the production and financial income loss.

Modern industry has widely used the fast acting and high reliable techniques for the maintenance and diagnosing the faults in the system, such that the techniques can reduce the unexpected failures and down time. It's essential that the operation of the machine in unsafe condition must be avoided. Nevertheless failures are unavoidable, and statistics of failures [8] in the induction motor components are mentioned as follows. A statistical study by the Electric Power Research Institute (EPRI) provides that bearing faults of $41 \%$, stator faults of $37 \%$, rotor faults of $10 \%$ and other faults of $12 \%$ [8]. It's important to spot the stator winding related faults as they are making up to $38 \%$ of all the faults, else they can lead to the total destruction of motor.
Therefore, reliable system with continuous monitoring is essential for the detection or diagnosing of such type of faults at early stage.

The present work is devoted to fault diagnosis of induction motor drive on its stator side using fuzzy logic approach. In this approach, the fault is diagnosed based on the knowledge from the rules which are generated in rule base. This method has been chosen because fuzzy logic has proved that it has ability in resembling the human decisions, and different stator faults can be easily monitored by using this technique.

In this paper, the fuzzy logic controller will diagnose different faults such as double line fault and unbalanced fault which are created on the stator side of an induction motor over a period of duration. Circuit Model is simulated using MATLAB ${ }^{\circledR}$ SIMULINK and simulation results are presented.

\section{ANALYSIS OF DIFFERENT STATOR FAULTS}

In three phase induction motors, there are different stator side winding faults which are as follows [11]

- $\quad$ Phase to Phase short fault

- Phase to earth short fault

- Open circuit in one phase

- Turn to turn short in a coil

From the above mentioned faults inter turn fault [8] is the initial process of the fault and it will lead to the different severe faults such as phase to phase fault, open circuit fault and etc. In general, motor current signature analysis [8] is used for the diagnosing these faults, whereas in this current paper different faults such as double line to ground fault and the unbalanced fault are created and diagnosed by using fuzzy logic in the system.

Under healthy condition, there is a balanced three phase currents in all the three phases. During the period of fault, these balanced three phase currents become unbalanced three phase currents, and this results in open circuit or leads to another severe phase faults. The main idea in creating the unbalance in the proposed system is for the fast monitoring of fault and avoiding a major motor failure. The system is also having the capability to detect the phase faults as well as the open circuit faults. 


\section{BLOCK DIAGRAM FOR THE PROPOSED SYSTEM}

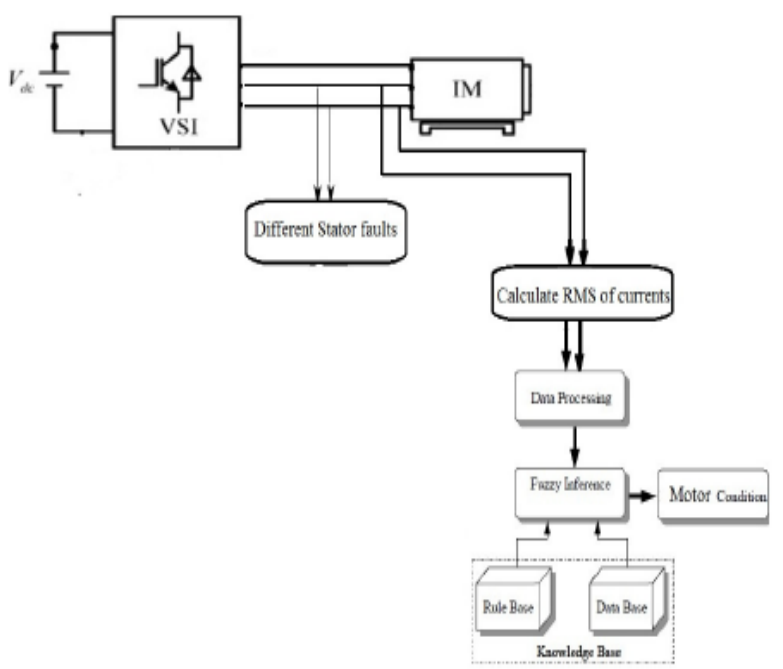

Fig. 1. Block diagram of the proposed system

Block diagram of voltage source inverter fed induction motor drive and fuzzy logic approach fault detection system is shown in Figure 1. The above system basically consists of a voltage source inverter fed induction motor. Under unhealthy condition such as during unbalanced or phase fault or open circuit fault, the system monitors the current amplitudes of the phases, and the root mean square (rms) value of each phases is calculated over the period. The rms value over a period of time is given by

$$
\text { rms value }=\sqrt{\left(\frac{1}{T}\right)} \int_{t}^{t+T} I(t)^{2} d t
$$

Where I ( $\mathrm{t}$ ) is the input current and $\mathrm{T}$ is fundamental time period. In the layout shown above, the stator rms values of currents are considered as the input variables to the fuzzy system, and motor condition such as which type of fault is going to be diagnosed by using this approach. The stator condition can be known by observing the current amplitudes in relation with stator condition. Numerical data can be represented as linguistic information.

\section{FUZZY DETECTION SYSTEM}

Fuzzy detection system essentially consists of data processing where the RMS values are used as inputs to the fuzzy system, and based on the knowledge acquisition and rule base, inference system finally decides the status of stator condition i.e., which type of fault occurred on the stator side can be diagnosed using this system.

\subsection{Fuzzy System Input \& Output Variables}

The amplitudes of currents are represented as input linguistic information. These categories are Zero (Z),Small (S) Medium (M), Large (L), Very large (VL) whereas the output linguistic variables are categorized by using Damaged (D), Unbalance (U), Healthy (H), Open circuit (O), Double Line (DL), where ' $U$ ' represents the unbalance currents in the phases, ' $H$ ' represents the stator with no fault ,'O' represents the open circuit fault in phase A. 'DL" represents the double line faults i.e. phase to phase faults. 'O' represents the open circuit fault in phase B or phase C. Using fuzzy set theory, the input and output of the system are represented as fuzzy sets The inputs and output of the system are represented as

$$
\begin{array}{r}
\boldsymbol{I}_{\boldsymbol{b}}=\left\{\boldsymbol{\mu}_{\boldsymbol{I} \boldsymbol{b}} \boldsymbol{I}_{\boldsymbol{b j}} / \boldsymbol{I}_{\boldsymbol{b j}} \in \boldsymbol{I}_{\boldsymbol{b}}\right\} \\
\boldsymbol{I}_{\boldsymbol{c}}=\left\{\boldsymbol{\mu}_{I c} \boldsymbol{I}_{\boldsymbol{c} j} / \boldsymbol{I}_{\boldsymbol{c} j} \in \boldsymbol{I}_{\boldsymbol{c}}\right\} \\
\boldsymbol{S C}=\left\{\boldsymbol{\mu}_{\boldsymbol{s c}} \boldsymbol{S} \boldsymbol{C}_{\boldsymbol{j}} / \boldsymbol{S} \boldsymbol{C}_{\boldsymbol{j}} \in \boldsymbol{S C}\right\}
\end{array}
$$

$I_{b}, I_{c}, S C$ are the universe of discourse. $I_{b} j, I_{c} j, S C j$ are the elements of universe of discourse. $\mu_{\mathrm{Ib}}, \mu_{\mathrm{Ic}}, \mu_{\mathrm{sc}}$ are the corresponding membership functions.

\subsection{Membership Functions}

Based on the rms data obtained under different fault conditions, the membership functions and corresponding limits are assigned for both input linguistic variables and the output linguistic variables. It was found that the combination of trapezoidal and triangular membership function is most suitable for the fault diagnosis of induction motors [3]. Fuzzy membership functions for the both input linguistic variables and output linguistic variables are shown below.

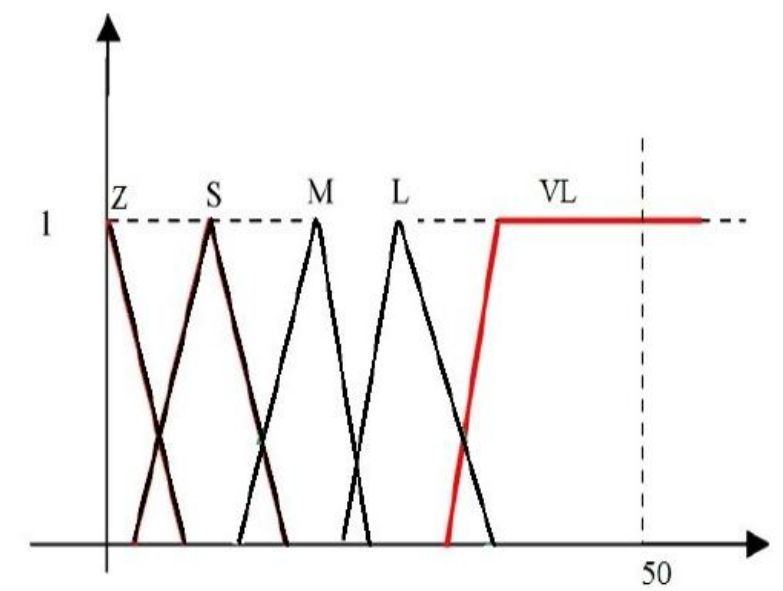

Fig. 2. Membership functions for the input variables

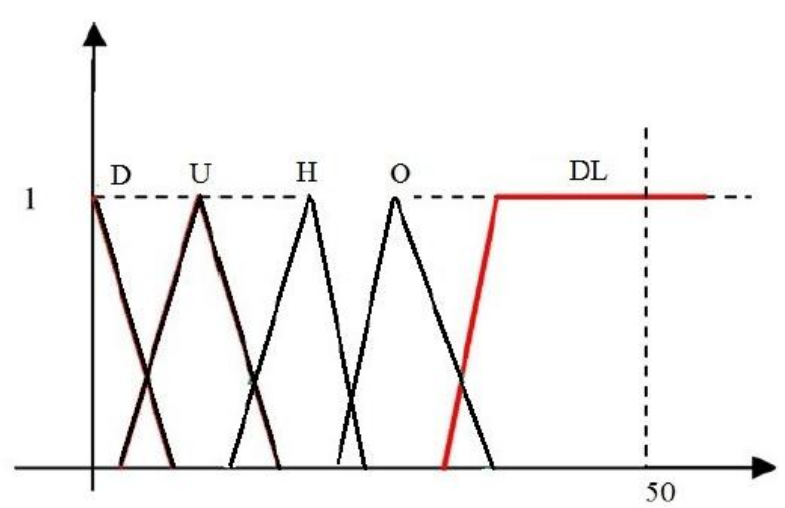

Fig 3. Membership functions for the output variables 
Fig 2.shows the membership function for the input linguistic variables where the stator two phase currents $I_{b}, I_{c}$ are the inputs to the fuzzy system and the output stator condition with Damaged (D), Unbalance (U), Healthy (H), Open circuit $(\mathrm{O})$ and Double line (DL) linguistic variables are shown in above Figure 3. Here in this instant limits for input as well as output are considered as same. The limits are chosen based on the rms values under healthy and faulty case.

\subsection{Fuzzy Inference System}

In this case rules are framed based on the knowledge obtained from the system simulated. Fuzzy inference system essentially consists of getting knowledge and the formation of rules.

Knowledge acquisition initiate with motor understanding if then rules. Under healthy condition the rotor rms values of currents are equal with a tolerance value and this is equivalent to say in rule 13.Under faulty case such as open circuit in phase ' $a$ ' is equivalent to say in rules 16-20, Double line fault in the system can be equivalent to say in the rules 21-25, unbalance in the system is equivalent to say in the rules 7-10. Based on all possible combinations with two currents and five linguistic variables a total of 25 rules are framed. This set of rules contains knowledge and gives the machine condition. Fuzzy rule table is shown below in Table 1 .

\begin{tabular}{|l|l|l|l|l|l|}
\hline \multicolumn{1}{|c|}{$\mathbf{I}_{\text {brms }}$} & Zero & Small & Medium & Large & Very large \\
\hline Zero & Damaged & Damaged & Damaged & Damaged & Damaged \\
\hline Small & Damaged & Unbalanced & Unbalanced & Unbalanced & Unbalanced \\
\hline Medium & Damaged & Healthy & Healthy & Open circuit & LLG \\
\hline Large & Damaged & Open circuit & Open circuit & Open circuit & LLG \\
\hline $\begin{array}{l}\text { Very } \\
\text { large }\end{array}$ & Damaged & LLG & LLG & LLG & LLG \\
\hline
\end{tabular}

In the final stage, the fuzzy values are converted into crisp ones using centroid method. In this method, affected membership is cut at a level of previous max rule, and then center of gravity of possible distribution is computed and becomes the numerical output value [7].The algebraic expression for centroid method is given below [7].

$$
z^{*}=\frac{\int \mu_{c}(Z) \cdot Z d z}{\int \mu_{c}(Z) \cdot d z}
$$

Where $Z^{*}$ is the defuzzified value, and $\int$ denotes algebraic integration.

\section{SIMULATION CIRCUITS AND RESULTS}

Fig. 4 shows the Matlab Simulink diagram of three phase voltage source inverter (VSI) fed induction motor drive under healthy condition.VSI is operated under 180 degree mode of operation.

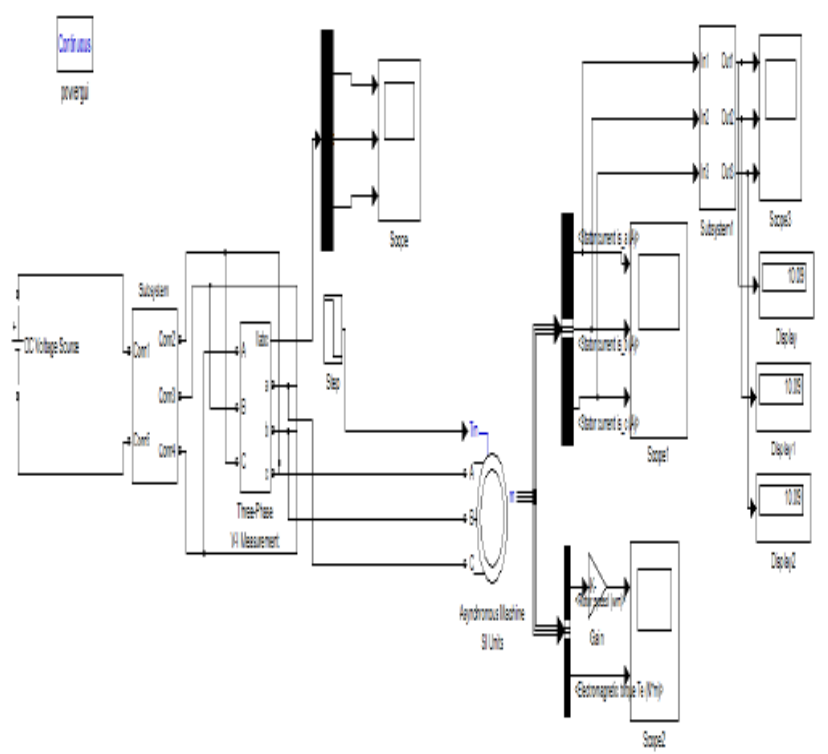

Fig. 4. Healthy motor simulation

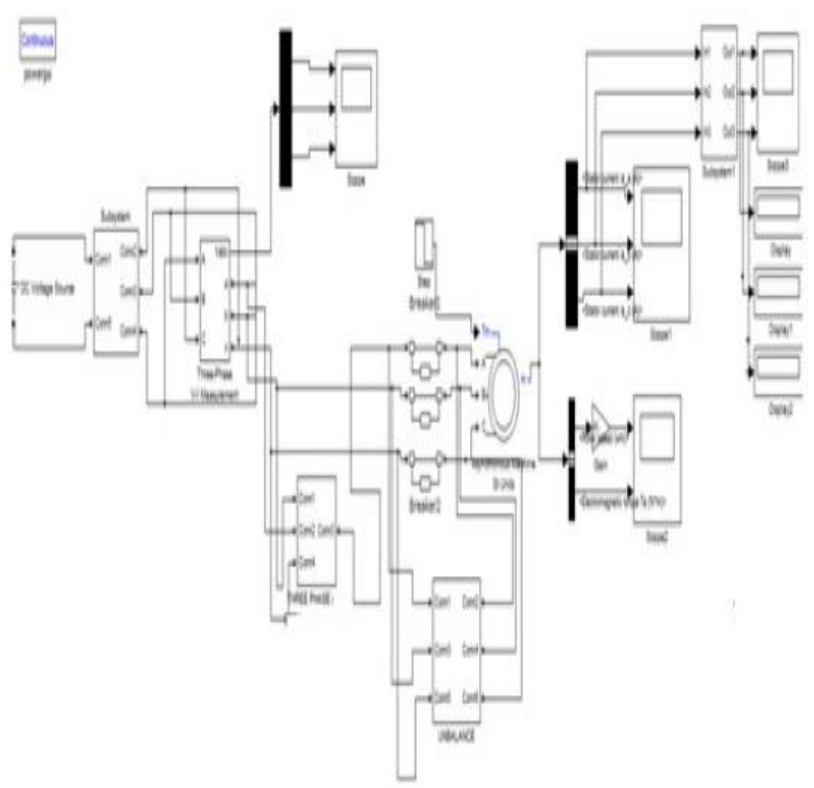

Fig 5.Simulation circuit implementing different faults

Fig.5. Shows the Matlab simulation diagram of double line to ground fault and three phase unbalance fault incorporated in the three phase VSI fed induction motor drive. Double line to ground fault is created in the above system between 2 and 3 seconds where as three phase unbalanced fault is created between 7 and 8 seconds. 


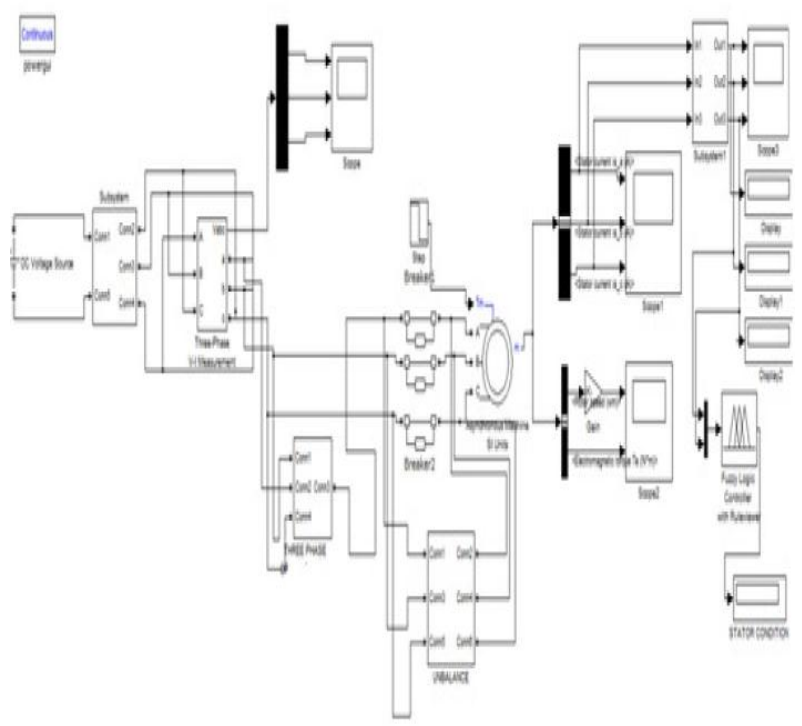

Fig 6. Fuzzy logic fault detection

Fuzzy logic controller is used to detect the various conditions, such as healthy state, double line to ground fault and three phase unbalanced fault, of the three phase VSI fed induction motor drive.

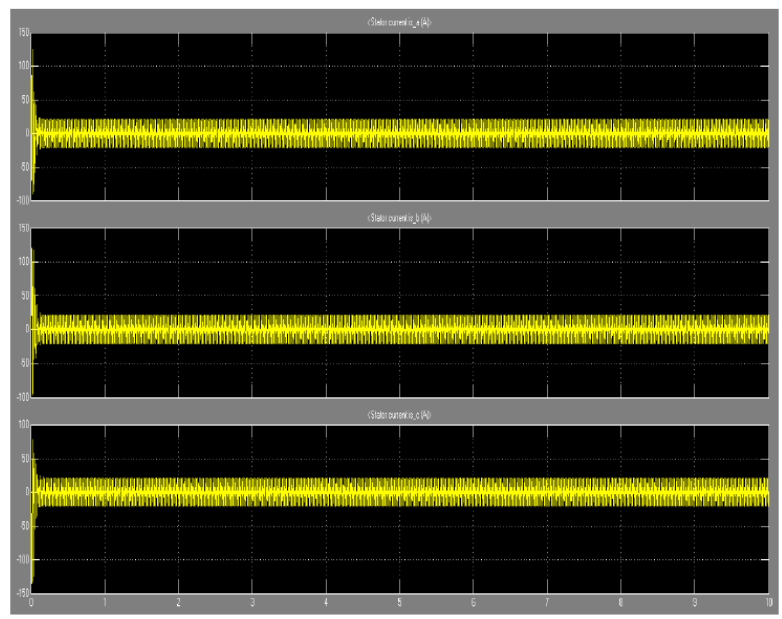

Fig 7. Stator currents of IM under healthy condition

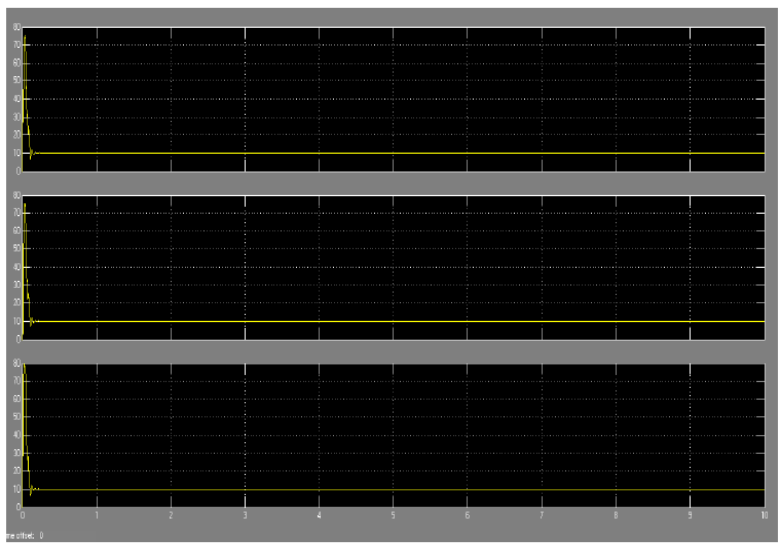

Fig 8. RMS stator currents of IM under healthy condition

Stator three phase currents under healthy condition are shown in the Fig 7. RMS values of three phase stator currents of induction motor are shown in the above figure. It is understood from figure 8 that the currents of phase A,B and $\mathrm{C}$ are in equal value for the healthy state of the drive.

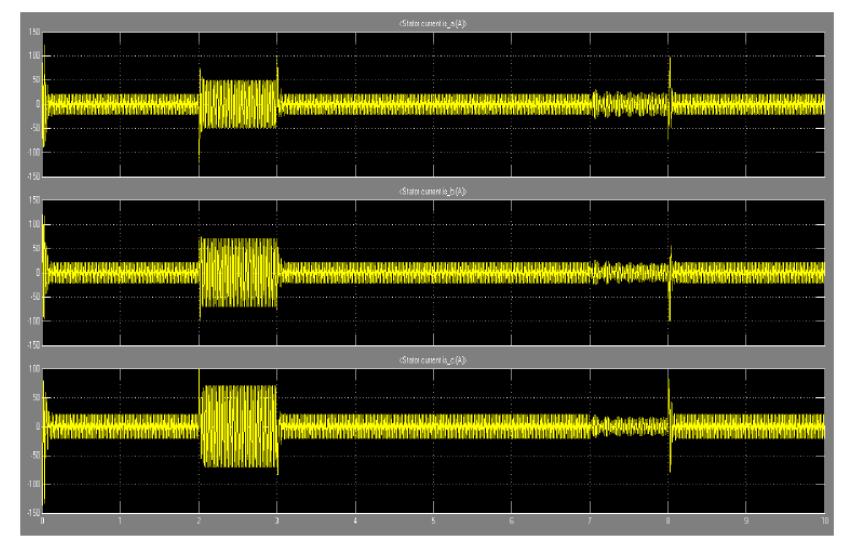

Fig 9. Stator currents under different faulty condition

Simulated stator currents of induction motor for the double line to ground fault and three phase unbalanced fault of induction motor drive is shown in Fig. 9

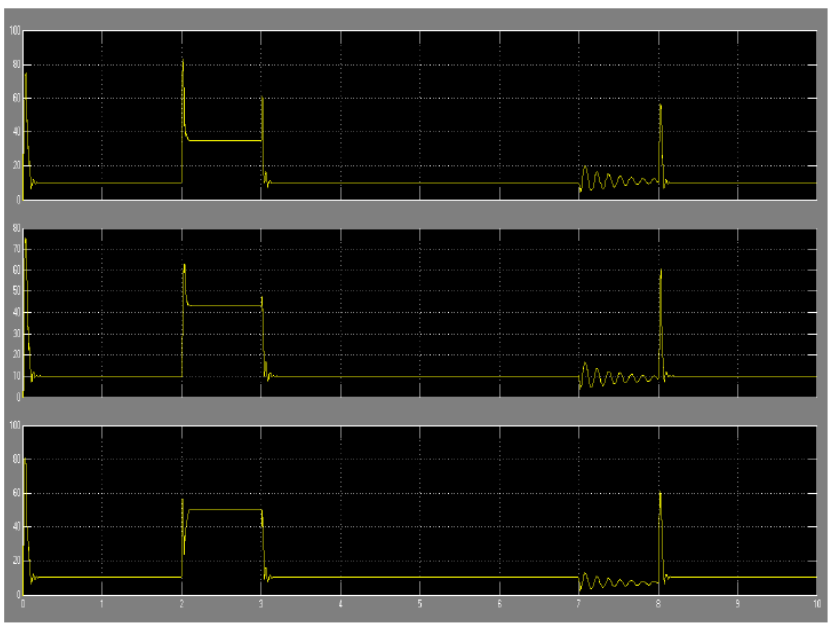

Fig 10.RMS Stator currents under different faulty

RMS current waveform of three phase stator currents of induction motor drive for double line to ground fault and three phase unbalanced fault are shown in the above Figure 10. Between 2 and 3 seconds, phase $\mathrm{B}$ and phase $\mathrm{C}$ currents are almost equal whereas phase $\mathrm{A}$ current has lesser magnitude than phase $\mathrm{B}$ and $\mathrm{C}$ currents because of double line to ground fault. Between 7 and 8 seconds, phase A, B and C currents have different magnitudes because of three phase unbalanced fault. 


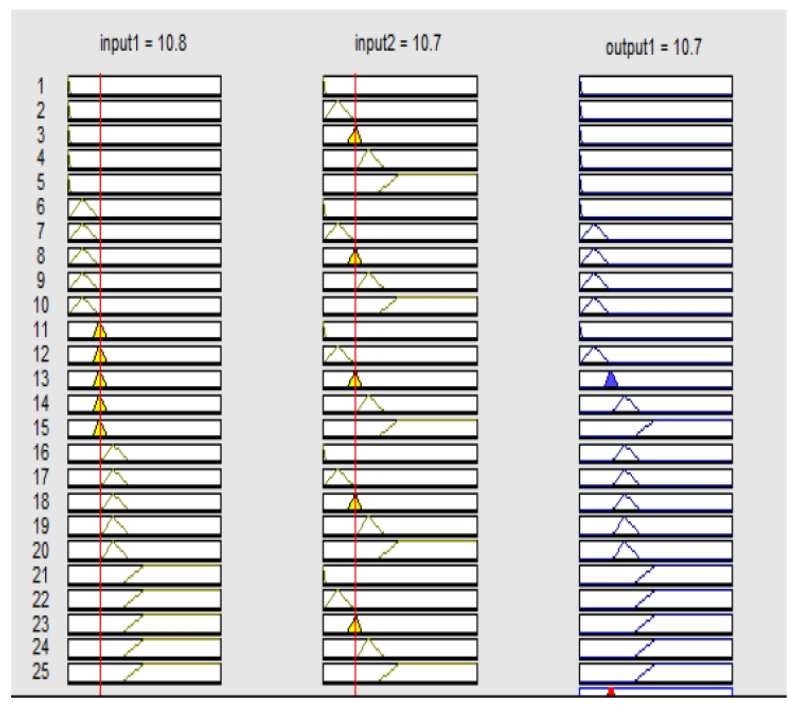

Figure 11.Fuzzy inference diagram under healthy condition

The RMS values of stator currents are applied to the fuzzy logic controller and the corresponding fuzzy logic rule viewer under different motor conditions are shown in the Figures 1113. Figure 11 Shows the result of fuzzy detection circuit under healthy condition with an output value of 10 based on the input stator currents.

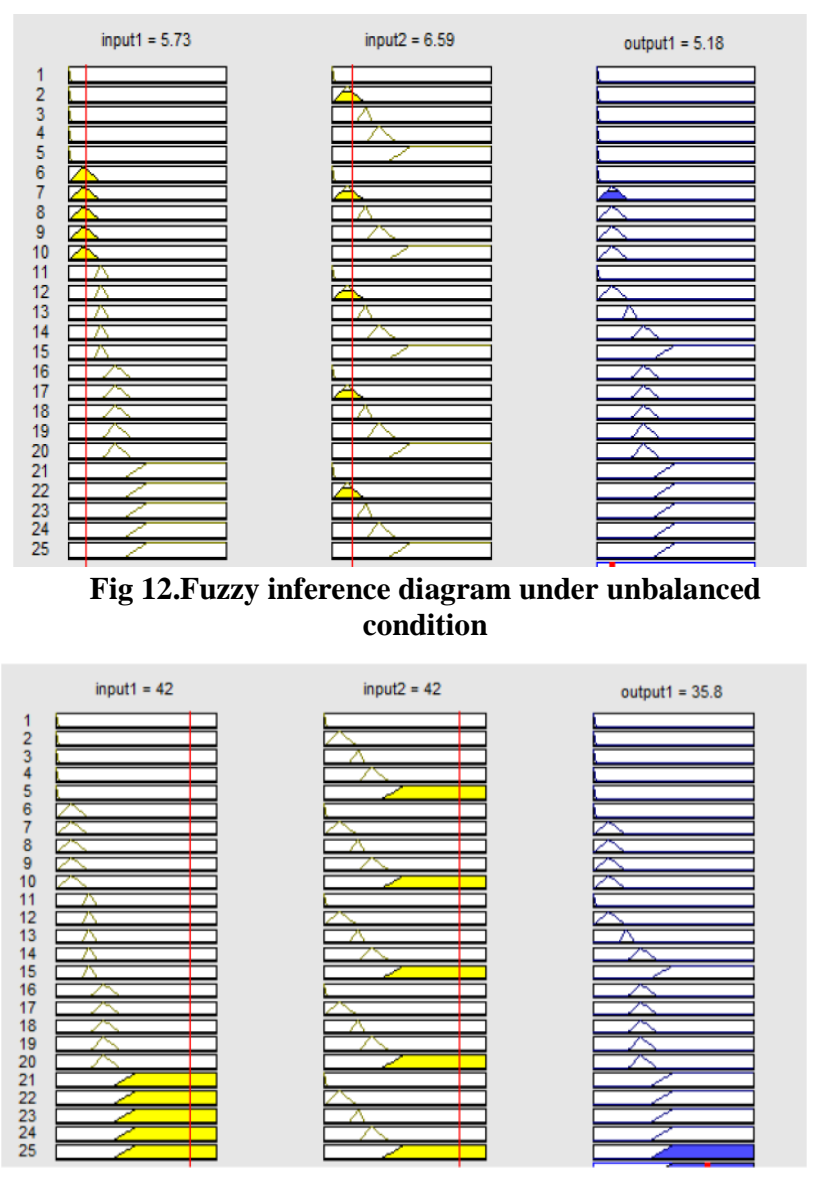

Figure 12 and Figure 13 shows the fuzzy inference diagram under unbalanced and double line to ground fault condition respectively. The motor condition is decided according to the output value and varies according to different fault conditions.

\section{CONCLUSION}

In this paper, three phase VSI fed induction motor under healthy condition, unbalanced fault and double line to ground fault are simulated using fuzzy controller. Unbalanced fault is created between 7 and 8 seconds, and double line to ground fault is created between 2 and 3 seconds. Fuzzy logic controller helps in diagnosing various conditions such as healthy state, double line to ground fault and unbalanced fault of three phase VSI fed induction motor drive.

\section{REFERENCES}

[1] M.E.H. Benbouzid, 2001. A Simple Fuzzy Logic Approach for Induction Motors Stator Condition Monitoring" in: Electric Machines and Drives Conference.

[2] X.Z. Gao, S.J. Ovaska, 2001. Soft Computing Methods for Control and Instrumentation. Thesis for the Degree of Doctor of Science and Technology, Helsinki.

[3] F. Filippetti et al, 1988. An approach to knowledge representation about induction machine diagnostics in expert systems Proceedings of ICEM.

[4] Z. Ye et al, 2001. Simulation of Electrical faults of three phase Induction motor drive system. in CD-ROM Proceedings of IEEE-PESC

[5] Mini.V.P, Dr.S.usha Kumari, 2010. Fault detection and diagnosis of induction motor using fuzzy logic. IEEE Region 8 SIBIRCON-2010, Irkutsk Listvyanka, Russia.

[6] G.M. Joksimovic, J. Penman, 2000. The detection of inter-turn short circuits in the stator windings of operating motors. IEEE Trans. Industrial Electronics 47 (5) $1078-2000$.

[7] Timothy J.Ross. "Fuzzy logic with engineering applications" pages 101-103

[8] W.T.Thomas, M.fenger, 2001. Current signature analysis to detect induction motor faults. IEEE Trans.Ind .Appl.

[9] F. Filippetti et al, I995. A fuzzy logic approach to on-line induction motor diagnostics based on stator current monitoring Proceedings of IEEE International Power Tech Conference

[10] Fernando Briz, Michael W. Degner, Juan M. Guernero and Pablo Garcia, "Stator windings fault diagnostics of induction machines operated from inverters and softstarters using high frequency negative sequence currents," IEEE transactions on industry applications, vol.45, 1637-1646, September / October 2009.

[11] B. E. H. Benbouzid, "A review of induction motors signature analysis as a medium for faults detection", IEEE Trans. Industrial Electronics, vol. 47, no. 5, pp. $984-993$, Oct. 2000.

Fig 13.Fuzzy inference diagram under LLG condition 